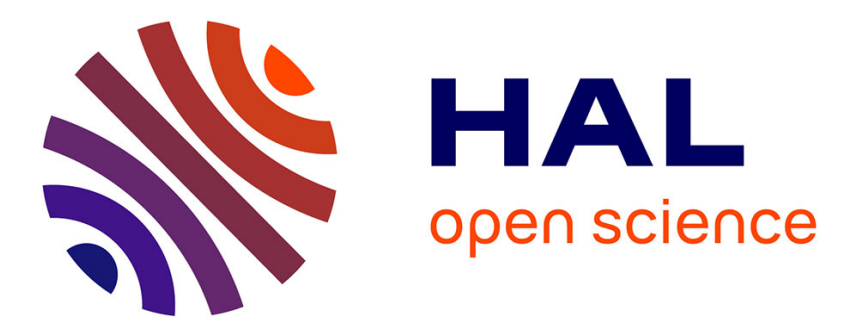

\title{
Robust output feedback control: convex lifting based approach
}

\author{
Ngoc Anh Nguyen, Sorin Olaru
}

\section{To cite this version:}

Ngoc Anh Nguyen, Sorin Olaru. Robust output feedback control: convex lifting based approach. 56th IEEE Conference on Decision and Control (CDC 2017), Dec 2017, Melbourne, Australia. pp.23302335, 10.1109/cdc.2017.8263990 . hal-01692255

\section{HAL Id: hal-01692255 \\ https://hal-centralesupelec.archives-ouvertes.fr/hal-01692255}

Submitted on 24 Jan 2018

HAL is a multi-disciplinary open access archive for the deposit and dissemination of scientific research documents, whether they are published or not. The documents may come from teaching and research institutions in France or abroad, or from public or private research centers.
L'archive ouverte pluridisciplinaire HAL, est destinée au dépôt et à la diffusion de documents scientifiques de niveau recherche, publiés ou non, émanant des établissements d'enseignement et de recherche français ou étrangers, des laboratoires publics ou privés. 


\title{
Robust output feedback control: convex lifting based approach
}

\author{
Ngoc Anh Nguyen ${ }^{1}$, Sorin Olaru ${ }^{1}$
}

\begin{abstract}
This paper presents a design method for robust output feedback control of linear, discrete-time, invariant systems affected by both state and output additive disturbances. The method relies on the so-called convex lifting, which is defined on the $N$-step controllable set. It is proven that the proposed method guarantees the recursive feasibility and robust stability in the sense that the closed loop converges to a given robust positively invariant set as time tends to infinity. Moreover, the method only requires the resolution of a linear programming problem at each sampling instant. Finally, a numerical example is considered to illustrate the methodology.
\end{abstract}

\section{INTRODUCTION}

Robust control is still an active topic in control theory, although extensive studies have been dedicated to over several decades, see among the others [16], [27]. In these robust control problems, full knowledge of the state is required, however, in practice measurement is usually affected by external noises. Therefore, suitable observer is required to estimate the state and to integrate in control design. As a consequence, control strategy becomes output feedback, since it is a function of outputs. Among the available methods, a basic idea is to transform a robust output feedback control problem into a robust state feedback counterpart, see [6]. Accordingly, robust control methodologies can be applied. Note that if the underlying system is linear and subject to linear constraints, a linear Luenberger observer can be deployed. On this direction, model predictive control (MPC) strategies were proposed in [14], [26]. A common point of MPC strategies is that suitable terminal constraints, describing a robust positively invariant set, are imposed at the end of the prediction horizon, leading to robust stability [15]. However, this also leads to an exponential increase of the number of constraints along the prediction horizon and subsequently to a demanding online evaluation.

To reduce the computational complexity, set-theoretic methods can also be of use by determining a priori a suitable control Lyapunov function, as employed in [4]. This method only requires to solve a linear program at each sampling instant. However, it also requires the contractivity of the feasible region over which this control Lyapunov function is defined. The computation of such a contractive set is usually based on an iterative procedure, known to be demanding. Recall that the vertex control presented in [10] also requires the contractivity of the feasible region. As an extension, the

Ngoc Anh Nguyen, Sorin Olaru are with the Laboratory of Signals and Systems (L2S, UMR CNRS 8506), CentraleSupélec-CNRS-UPS, Université Paris Saclay, 3 rue Joliot Curie, Plateau de Moulon, 91192, Gif-sur-Yvette, France. Ngocanh.Nguyen.rs@gmail.com, Sorin.Olaruecentralesupelec.fr interpolation based approach was put forward in [18], in which the interpolation gain serves as a control Lyapunov function. Accordingly, robust stability is ensured by the nonincrease of this gain along the dynamics. Moreover, the socalled convex lifting approach has recently been presented in [22], [24]. Unlike the above set-theoretic methods, this concept is not a control Lyapunov function, however the contractivity of the feasible region is still required.

This paper presents an alternative method, relying on a suitable convex lifting as an extension of the method in [22], [24]. More precisely, this convex lifting is defined over an $N$-step controllable set, known not to be contractive. Similar to these set-theoretic methods, terminal constraints are not imposed. However, unlike a control Lyapunov function, this function is equal to 0 over a given robust positively invariant set and strictly positive outside this region. In addition, this function is shown to be strictly decreasing along the closed-loop dynamics outside this invariant set, leading to its convergence to 0 as time tends to infinity. Accordingly, robust stability is guaranteed by means of the convergence of the closed-loop dynamics to a given robust positively invariant set. Meanwhile, this method also requires to solve a linear program at each sampling instant, it thus could be useful for systems with fast dynamics, e.g. cantilever beam system [9].

\section{Nomenclature}

Throughout the paper, $\mathbb{R}, \mathbb{N}, \mathbb{N}_{>0}$ denote the field of real numbers, the set of nonnegative integers and the positive integer set, respectively. The index set $\mathcal{I}_{N}$ is also defined as $\mathcal{I}_{N}=\{1,2, \ldots, N\}$ with respect to a given $N \in \mathbb{N}_{>0}$. A polyhedron is defined as the intersection of finitely many closed halfspaces. A polytope is defined as a bounded polyhedron. Also, $\mathcal{V}(P)$ denotes the set of vertices of polytope $P$. We use $\operatorname{int}(S)$ to denote the interior of a full-dimensional set $S$ and $\operatorname{conv}(S)$ denotes the convex hull of $S$. Also, given a set $S$ and a matrix $A$ of suitable dimension, we use $A S$ to denote the following set: $A S=\{A x: x \in S\}$. Given two sets $S_{1}, S_{2} \subset \mathbb{R}^{d}$, by $S_{1} \backslash S_{2}$, we denote the following set: $S_{1} \backslash S_{2}:=\left\{x \in \mathbb{R}^{d}: x \in S_{1}, x \notin S_{2}\right\}$. Also, the Minkowski sum of these two sets, denoted by $S_{1} \oplus S_{2}$, is defined as follows: $S_{1} \oplus S_{2}:=\left\{x_{1}+x_{2}: x_{1} \in S_{1}, x_{2} \in S_{2}\right\}$. Finally, the Potryagin difference $S_{1} \ominus S_{2}$ is defined by: $S_{1} \ominus S_{2}:=\left\{x: x \oplus S_{2} \subseteq S_{1}\right\}$.

\section{PRoblem Settings}

In this paper, we consider a linear time-invariant system, affected by both additive state and output disturbances:

$$
\begin{aligned}
x_{k+1} & =A x_{k}+B u_{k}+w_{k} \\
y_{k} & =C x_{k}+v_{k}
\end{aligned}
$$


where $x_{k}, u_{k}$ denote the state and control variable at time $k$, while $w_{k}, v_{k}$ represent the additive state and output disturbances at time $k$, respectively. These variables and disturbances satisfy:

$$
x_{k} \in \mathbb{X}, u_{k} \in \mathbb{U}, w_{k} \in \mathbb{W}, v_{k} \in \mathbb{V},
$$

where the constraint sets $\mathbb{X} \subset \mathbb{R}^{d_{x}}, \mathbb{U} \subset \mathbb{R}^{d_{u}}, \mathbb{W} \subset \mathbb{R}^{d_{x}}, \mathbb{V} \subset$ $\mathbb{R}^{d_{y}}$ are assumed to be polytopes, containing the origin in their interior, with given $d_{x}, d_{u}, d_{y} \in \mathbb{N}_{>0}$. This system is assumed to satisfy the following assumption.

Assumption 1: The pair $(A, B)$ is controllable and the pair $(A, C)$ is observable.

This paper aims to design robust output feedback controller which is able to cope with the state and output disturbances, and to stabilize system (1), while guaranteeing the constraints in (2). To tackle this problem, a common approach is to make use of a Luenberger observer. More precisely, this observer is written in the following form:

$$
\hat{x}_{k+1}=A \hat{x}_{k}+B u_{k}+L\left(y_{k}-\hat{y}_{k}\right), \hat{y}_{k}=C \hat{x}_{k} .
$$

If one defines $e_{k}=x_{k}-\hat{x}_{k}$, then we obtain the following autonomous system, according to (1) and (3),

$$
e_{k+1}=(A-L C) e_{k}+w_{k}-L v_{k}
$$

Note that since $w_{k} \in \mathbb{W}$ and $v_{k} \in \mathbb{V}$, then $w_{k}-L v_{k}$, considered as additive disturbances of system (4), is also bounded in $\mathbb{W} \oplus(-L \mathbb{V})$, i.e., $w_{k}-L v_{k} \in \mathbb{W} \oplus(-L \mathbb{V})$. Therefore, if one chooses a gain $L$ such that $A-L C$ is strictly stable, then it is proven in [8] that a robust positively invariant set exists (this concept will be recalled in Sect. III). Such a gain $L$ can be determined by means of the Riccati equation for the system $\left(A^{T}, C^{T}\right)$.

For ease of presentation, let $\Omega_{e}$ denote a robust positively invariant set for the autonomous system (4). Accordingly, for any initial error state $e_{0} \in \Omega_{e}$, then $e_{k} \in \Omega_{e}$ for all $k \in \mathbb{N}_{>0}$. The observer system (3) can be written in the following form:

$$
\hat{x}_{k+1}=A \hat{x}_{k}+B u_{k}+L v_{k}+L C e_{k}, \hat{y}_{k}=C \hat{x}_{k} .
$$

We remark that if the initial error state $e_{0} \in \Omega_{e}$, then $L v_{k}+L C e_{k}$ is also considered as additive disturbance for the observer system (5). Furthermore, such an additive disturbance is also bounded in $L \mathbb{V} \oplus L C \Omega_{e}$. Note that $\mathbb{X}, \mathbb{V}, \mathbb{W}$ are assumed to be polytopes, therefore $\Omega_{e}$ is bounded, leading to the boundedness of $L \mathbb{V} \oplus L C \Omega_{e}$. If we compute a polytopic robust positively invariant set $\Omega_{e}$ for the autonomous system (4), then we can ensure that the additive disturbance $L v_{k}+$ $L C e_{k}$ is bounded in polytope $L \mathbb{V} \oplus L C \Omega_{e}$. Also, it can easily be observed that $0 \in \operatorname{int}\left(L \mathbb{V} \oplus L C \Omega_{e}\right)$, since $0 \in \operatorname{int}(\mathbb{V})$ and $0 \in \operatorname{int}\left(\Omega_{e}\right)$. As a consequence, we now return to a robust control design problem for the linear system (5) affected by bounded additive state disturbances, whose variables and parameters are bounded as below:

$$
\hat{x}_{k} \in \mathbb{X} \ominus \Omega_{e}, u_{k} \in \mathbb{U}, L v_{k}+L C e_{k} \in L \mathbb{V} \oplus L C \Omega_{e},
$$

under the assumption that $\mathbb{X} \ominus \Omega_{e}$ is non-empty. In the sequel, we will present a robust control design method for system
(5), which relies on a suitable convex lifting. Unlike the counterpart presented in [22], [24], a convex lifting employed in this paper is defined over the $N$-step controllable set, usually known not to be contractive. Note that the contractivity property is always required in most of existing methods related to piecewise linear control Lyapunov functions [4], [10], [17]. It is worth emphasizing that robust stability in this paper is not in the sense of Lyapunov. Instead, we will prove that such a constructive convex lifting is strictly decreasing along the closed-loop dynamics outside a given robust positively invariant set and consequently the closed loop of the observer system (5) is convergent to this set, leading to robust stability of system (1).

\section{CONSTRUCTION OF SUITABLE CONVEX LIFTING}

Before going to the main result, we recall some important concepts which are of use later in the proposed control design method. Positive invariance concept has been investigated in many studies [1]-[3], [5] and deployed in different control design strategies. In case the underlying system is affected by disturbances, the robust positive invariance concept is of use instead.

Definition 3.1: Given an admissible control law $u=$ $K \hat{x} \in \mathbb{U}$, a set $\Omega_{\hat{x}} \subseteq \mathbb{X} \ominus \Omega_{e}$ is called robust positively invariant with respect to system (5) subject to constraints (6) iff $(A+B K) \Omega_{\hat{x}} \oplus L \mathbb{V} \oplus L C \Omega_{e} \subseteq \Omega_{\hat{x}}$.

Similar to determining an observer gain, such a local controller $u=K \hat{x} \in \mathbb{U}$ in Definition 3.1 can also be obtained via the Riccati equation for the pair $(A, B)$. According to this local controller, one can use existing algorithms to compute a robust positively invariant set $\Omega_{\hat{x}}$, see for instance [8], [13], [17], [25]. Hereafter, $\Omega_{\hat{x}}$ is assumed to be a full-dimensional polytope.

Another important concept is the feasible region. Unlike the one deployed in [24], in this paper we make use of the $N$-step controllable set as the feasible region with respect to a given $N \in \mathbb{N}_{>0}$. Its definition is recalled below.

Definition 3.2: Consider system (5) subject to constraints (6). Let a robust positively invariant set $\Omega_{\hat{x}}$ and $N \in \mathbb{N}_{>0}$ be given. A set denoted by $\mathcal{K}_{N}\left(\Omega_{\hat{x}}\right) \subseteq \mathbb{X} \ominus \Omega_{e}$ is called the $N$-step controllable set if any point, belonging to this set, can reach $\Omega_{\hat{x}}$ in $N$ steps, while staying inside $\mathbb{X} \ominus \Omega_{e}$ despite any disturbances in $L \mathbb{V} \oplus L C \Omega_{e}$, i.e.,

$$
\begin{aligned}
\mathcal{K}_{0}\left(\Omega_{\hat{x}}\right)= & \Omega_{\hat{x}}, \\
\mathcal{K}_{N}\left(\Omega_{\hat{x}}\right)= & \left\{\hat{x}_{0} \in \mathbb{X} \ominus \Omega_{e}: \exists u_{0}, \ldots, u_{N-1} \in \mathbb{U}\right. \text { s.t. } \\
& \left.\hat{x}_{N} \in \Omega_{\hat{x}}, \forall v_{i} \in \mathbb{V}, e_{i} \in \Omega_{e}, i \in\{0\} \cup \mathcal{I}_{N-1}\right\} .
\end{aligned}
$$

For brevity, the computation of $\mathcal{K}_{N}\left(\Omega_{\hat{x}}\right)$ is referred to [12] for further details. We now present the construction of a suitable function according to the above ingredients, which will be deployed in the proposed control design. For ease of presentation, let $\ell_{N}(\hat{x})$ denote such a function defined over $\mathcal{K}_{N}\left(\Omega_{\hat{x}}\right)$. According to its construction presented in the sequel, $\ell_{N}(\hat{x})$ was proven in [19], [24] to be a convex lifting, therefore, we will call this function a convex lifting 
throughout the rest of the paper. For simplicity, the formal definition of this concept and related ones are referred to [20], [21], [23]. This function should be convex, nonnegative, equal to 0 over $\Omega_{\hat{x}}$, strictly positive over $\mathcal{K}_{N}\left(\Omega_{\hat{x}}\right) \backslash \Omega_{\hat{x}}$. Furthermore, it should satisfy $\ell_{N}(v)>\max _{\hat{x} \in \mathcal{K}_{i-1}\left(\Omega_{\hat{x}}\right)} \ell_{N}(\hat{x})$ for all $v \in \mathcal{V}\left(\mathcal{K}_{i}\left(\Omega_{\hat{x}}\right)\right) \backslash \mathcal{K}_{i-1}\left(\Omega_{\hat{x}}\right)$. All these requirements are of use later to prove the convergence of $\ell_{N}(\hat{x})$ to 0 along the trajectories, as it will be clear later. To construct $\ell_{N}(\hat{x})$, we start with the following initial point, for $h_{0}=0$ and a given constant $h_{1}>0$ :

$$
\begin{aligned}
& V_{0}:=\left\{\left[v^{T} h_{0}\right]^{T}: v \in \mathcal{V}\left(\Omega_{\hat{x}}\right)\right\} \subset \mathbb{R}^{d_{x}+1} \\
& V_{1}:=\left\{\left[v^{T} h_{1}\right]^{T}: v \in \mathcal{V}\left(\mathcal{K}_{1}\left(\Omega_{\hat{x}}\right)\right)\right\} \cup V_{0} \\
& \Pi_{1}:=\operatorname{conv}\left(V_{1}\right) \\
& \ell_{1}(\hat{x}):=\min _{z} z \text { s.t. }\left[\hat{x}^{T} z\right]^{T} \in \Pi_{1} .
\end{aligned}
$$

The above function $\ell_{1}(\hat{x})$ represents the following properties.

Lemma 3.1: Function $\ell_{1}(\hat{x})$ is continuous, convex and piecewise affine.

The proof follows as a direct consequence of Theorems IV-3 and IV-4 in [7]. The following important properties of $\ell_{1}(\hat{x})$ are of use later.

Lemma 3.2: Function $\ell_{1}(\hat{x})$ satisfies:

1) $0 \leq \ell_{1}(\hat{x}) \leq h_{1}$ for all $\hat{x} \in \mathcal{K}_{1}\left(\Omega_{\hat{x}}\right)$;

2) $\ell_{1}(\hat{x})=0$ for $\hat{x} \in \Omega_{\hat{x}}$;

3) $\ell_{1}(\hat{x})>0$ for $\hat{x} \in \mathcal{K}_{1}\left(\Omega_{\hat{x}}\right) \backslash \Omega_{\hat{x}}$;

4) $\ell_{1}(\hat{x})=h_{1}$ for $\hat{x} \in \mathcal{V}\left(\mathcal{K}_{1}\left(\Omega_{\hat{x}}\right)\right) \backslash \Omega_{\hat{x}}$.

Proof: Any point $\left[\hat{x}^{T} z\right]^{T} \in \Pi_{1}$ can be expressed as:

$$
\begin{gathered}
\alpha_{0}(v), \alpha_{1}(v) \geq 0, \sum_{v \in \mathcal{V}\left(\Omega_{\hat{x}}\right)} \alpha_{0}(v)+\sum_{v \in \mathcal{V}\left(\mathcal{K}_{1}\left(\Omega_{\hat{x}}\right)\right)} \alpha_{1}(v)=1, \\
{\left[\begin{array}{l}
\hat{x} \\
z
\end{array}\right]=\sum_{v \in \mathcal{V}\left(\Omega_{\hat{x}}\right)} \alpha_{0}(v)\left[\begin{array}{l}
v \\
0
\end{array}\right]+\sum_{v \in \mathcal{V}\left(\mathcal{K}_{1}\left(\Omega_{\hat{x}}\right)\right)} \alpha_{1}(v)\left[\begin{array}{c}
v \\
h_{1}
\end{array}\right] .}
\end{gathered}
$$

Accordingly, one can easily observe that

$$
0 \leq z=\sum_{v \in \mathcal{V}\left(\Omega_{\hat{x}}\right)} \alpha_{0}(v) 0+\sum_{v \in \mathcal{V}\left(\mathcal{K}_{1}\left(\Omega_{\hat{x}}\right)\right)} \alpha_{1}(v) h_{1} \leq h_{1},
$$

leading to claim 1). Note that the left-hand inequality becomes equality only if $\alpha_{1}(v)=0$ for all $v \in \mathcal{V}\left(\mathcal{K}_{1}\left(\Omega_{\hat{x}}\right)\right)$, leading to $\hat{x} \in \Omega_{\hat{x}}$, as stated in claim 2). Moreover, for any point $\hat{x} \in \mathcal{K}_{1}\left(\Omega_{\hat{x}}\right) \backslash \Omega_{\hat{x}}$ expressed above, there exists at least one vertex $v \in \mathcal{V}\left(\mathcal{K}_{1}\left(\Omega_{\hat{x}}\right)\right)$ such that $\alpha_{1}(v)>0$, leading to $z>0$ and subsequently to claim 3$)$. Finally, since $\ell_{1}(\hat{x})$ is convex over $\mathcal{K}_{1}\left(\Omega_{\hat{x}}\right)$, this function attains its maximal value $h_{1}$ at vertices of $\mathcal{K}_{1}\left(\Omega_{\hat{x}}\right)$. Note however that any $\hat{x} \in \mathcal{V}\left(\mathcal{K}_{1}\left(\Omega_{\hat{x}}\right)\right) \cap \Omega_{\hat{x}}$ can be expressed as in (8) with $\alpha_{1}(v)=0$ for all $v \in \mathcal{V}\left(\mathcal{K}_{1}\left(\Omega_{\hat{x}}\right)\right)$, yielding $\ell_{1}(\hat{x})=0<h_{1}$. In other words, $\ell_{1}(\hat{x})=h_{1}$ for $\hat{x} \in \mathcal{V}\left(\mathcal{K}_{1}\left(\Omega_{\hat{x}}\right)\right) \backslash \Omega_{\hat{x}}$. In order to construct a convex lifting $\ell_{N}(\hat{x})$ as required above, we construct intermediate functions $\ell_{i}(\hat{x})$ for $i \in \mathbb{N}_{>0}$ such that $\ell_{N}(\hat{x})=\ell_{i}(\hat{x})$ for all $\hat{x} \in \mathcal{K}_{i}\left(\Omega_{\hat{x}}\right)$ and for all $i \in \mathcal{I}_{N}$. Starting from (7), the construction of $\ell_{N}(\hat{x})$ is presented step-by-step in the sequel. Suppose function $\ell_{i}(\hat{x})$, associated with the polytopic partition $\left\{\mathcal{X}_{j}^{(i)}\right\}_{j \in \mathcal{I}_{M^{(i)}}}$ of $\mathcal{K}_{i}\left(\Omega_{\hat{x}}\right)$, is written in the form:

$$
\ell_{i}(\hat{x})=\left(a_{j}^{(i)}\right)^{T} \hat{x}+b_{j}^{(i)} \text { for } \hat{x} \in \mathcal{X}_{j}^{(i)} .
$$

We also define the following function:

$$
\sigma_{i}(\hat{x})=\max _{j \in \mathcal{I}_{M^{(i)}}}\left(a_{j}^{(i)}\right)^{T} \hat{x}+b_{j}^{(i)} \text { for } \hat{x} \in \mathbb{R}^{d_{x}} .
$$

Accordingly, $\ell_{i+1}(\hat{x})$ is constructed as follows, with respect to a given constant $\epsilon>0$ :

$$
\begin{aligned}
& h_{i+1}:=\min _{h} h \text { s.t. } \sigma_{i}(v)+\epsilon \leq h, \forall v \in \mathcal{V}\left(\mathcal{K}_{i+1}\left(\Omega_{\hat{x}}\right)\right) \backslash \mathcal{K}_{i}\left(\Omega_{\hat{x}}\right) \\
& V_{i+1}:=\left\{\left[v^{T} h_{i+1}\right]^{T}: v \in \mathcal{V}\left(\mathcal{K}_{i+1}\left(\Omega_{\hat{x}}\right)\right)\right\} \cup V_{i} \\
& \Pi_{i+1}:=\operatorname{conv}\left(V_{i+1}\right)
\end{aligned}
$$

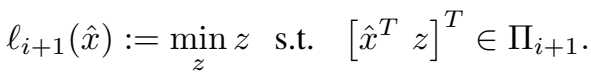

The constructions (9)-(11) are repeated until $i=N-1$. Similar to $\ell_{1}(\hat{x})$ shown in Lemma $3.1, \ell_{i+1}(\hat{x})$ for all $i \in$ $\mathcal{I}_{N-1}$ possess the following properties:

Lemma 3.3: Functions $\ell_{i+1}(\hat{x})$ are convex, continuous, piecewise affine, for all $i \in \mathcal{I}_{N-1}$.

Lemma 3.4: $\sigma_{i}(\hat{x})=\ell_{i}(\hat{x})$ for all $\hat{x} \in \mathcal{K}_{i}\left(\Omega_{\hat{x}}\right), \forall i \in \mathcal{I}_{N}$.

Proof: Since $\ell_{i}(\hat{x})$ is a convex, piecewise affine function, proven in Lemma 3.3, it can be written as follows:

$$
\ell_{i}(\hat{x})=\max _{j \in \mathcal{I}_{M^{(i)}}}\left(a_{j}^{(i)}\right)^{T} \hat{x}+b_{j}^{(i)} \text { for } \hat{x} \in \mathcal{K}_{i}\left(\Omega_{\hat{x}}\right) .
$$

Accordingly, the representation of $\sigma_{i}(\hat{x})$ in (10) leads to $\sigma_{i}(\hat{x})=\ell_{i}(\hat{x})$ for all $\hat{x} \in \mathcal{K}_{i}\left(\Omega_{\hat{x}}\right)$.

We now prove that function $\ell_{N}(\hat{x})$ satisfies the aforementioned requirements.

Lemma 3.5: For $i \in \mathcal{I}_{N}$, we obtain

1) $0<h_{i}<h_{i+1}$

2) $\ell_{i}(\hat{x})=h_{i}$ for $\hat{x} \in \mathcal{V}\left(\mathcal{K}_{i}\left(\Omega_{\hat{x}}\right)\right) \backslash \mathcal{K}_{i-1}\left(\Omega_{\hat{x}}\right)$

3) $0 \leq \ell_{i}(\hat{x}) \leq h_{i}$

4) $\ell_{i+1}(\hat{x})=\ell_{i}(\hat{x})$ for $\hat{x} \in \mathcal{K}_{i}\left(\Omega_{\hat{x}}\right)$.

Proof: We will prove claims 1), 2) and 3) in the same time. As proven in Lemma 3.2, for $i=1$, claims 2) and 3) hold true and $0<h_{1}$, we now prove that $h_{1}<h_{2}$. In fact, as $\sigma_{1}(\hat{x})$ is convex over $\mathcal{K}_{2}\left(\Omega_{\hat{x}}\right)$, it thus attains its maximal value at vertices of $\mathcal{K}_{2}\left(\Omega_{\hat{x}}\right)$. Accordingly, there exists a vertex $v \in \mathcal{V}\left(\mathcal{K}_{2}\left(\Omega_{\hat{x}}\right)\right) \backslash \mathcal{K}_{1}\left(\Omega_{\hat{x}}\right)$ such that $\sigma_{1}(v) \geq \sigma_{1}(\hat{x})$ for all $\hat{x} \in \mathcal{K}_{2}\left(\Omega_{\hat{x}}\right)$. Therefore, if one chooses $\hat{x} \in \mathcal{V}\left(\mathcal{K}_{1}\left(\Omega_{\hat{x}}\right)\right)$, then (11) yields $h_{2} \geq \epsilon+\sigma_{1}(\hat{x})=\epsilon+\ell_{1}(\hat{x})=\epsilon+h_{1}>h_{1}$.

To prove claim 3) for $i=2$, we consider $\left[\hat{x}^{T} z\right]^{T} \in \Pi_{2}$, this point can be described as below

$$
\begin{aligned}
& \alpha_{j}(v) \geq 0, \sum_{j=0}^{2} \sum_{v \in \mathcal{V}\left(\mathcal{K}_{j}\left(\Omega_{\hat{x}}\right)\right)} \alpha_{j}(v)=1 \\
& {\left[\hat{x}^{T} z\right]^{T}=\sum_{j=0}^{2} \sum_{v \in \mathcal{V}\left(\mathcal{K}_{j}\left(\Omega_{\hat{x}}\right)\right)} \alpha_{j}(v)\left[v^{T} h_{j}\right]^{T} .}
\end{aligned}
$$

As a consequence of claim 1) for $i=1$, we obtain

$$
0 \leq z=\sum_{j=0}^{2} \sum_{v \in \mathcal{V}\left(\mathcal{K}_{j}\left(\Omega_{\hat{x}}\right)\right)} \alpha_{j}(v) h_{j} \leq h_{2}
$$


leading to claim 3) for $i=2$.

To prove claim 2) holds true for $i=2$, consider any point $\left[\begin{array}{ll}\hat{x}^{T} z\end{array}\right]^{T} \in \Pi_{2}$, this can be written as in (12). Note that $\ell_{2}(\hat{x})$ is convex over $\mathcal{K}_{2}\left(\Omega_{\hat{x}}\right)$, therefore $\ell_{2}(\hat{x})$ attains its maximal value $h_{2}$ at vertices of $\mathcal{K}_{2}\left(\Omega_{\hat{x}}\right)$. However, any point $\hat{x} \in$ $\mathcal{V}\left(\mathcal{K}_{2}\left(\Omega_{\hat{x}}\right)\right) \cap \mathcal{K}_{1}\left(\Omega_{\hat{x}}\right)$ can be expressed as in form (12) with $\alpha_{2}(v)=0, \forall v \in \mathcal{V}\left(\mathcal{K}_{2}\left(\Omega_{\hat{x}}\right)\right)$, leading to $\ell_{2}(\hat{x}) \leq h_{1}<h_{2}$. Roughly speaking, $\ell_{2}(\hat{x})=h_{2}$ for $\hat{x} \in \mathcal{V}\left(\mathcal{K}_{2}\left(\Omega_{\hat{x}}\right)\right) \backslash \mathcal{K}_{1}\left(\Omega_{\hat{x}}\right)$. The proof of claims 1), 2) and 3) for $N \geq i \geq 3$ follows the same arguments as deployed above.

For claim 4), we first prove that it holds for $i=1$. In fact, as proven in claim 1) that $h_{2} \geq h_{1}+\epsilon$, then the construction in (11) leads to $h_{2} \geq \sigma_{1}(v)+\epsilon$ for all $v \in \mathcal{V}\left(\mathcal{K}_{2}\left(\Omega_{\hat{x}}\right)\right)$.

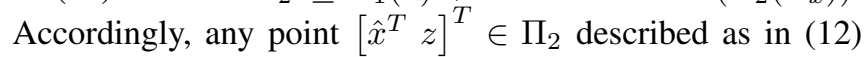
yields:

$$
\begin{aligned}
& z=\sum_{j=0}^{2} \sum_{v \in \mathcal{V}\left(\mathcal{K}_{j}\left(\Omega_{\hat{x}}\right)\right)} \alpha_{j}(v) h_{j} \\
& \geq \sum_{j=0}^{1} \sum_{v \in \mathcal{V}\left(\mathcal{K}_{j}\left(\Omega_{\hat{x}}\right)\right)} \alpha_{j}(v) \sigma_{1}(v) \\
& \quad+\sum_{v \in \mathcal{V}\left(\mathcal{K}_{2}\left(\Omega_{\hat{x}}\right)\right)} \alpha_{2}(v)\left(\sigma_{1}(v)+\epsilon\right) \\
& \quad \sum_{v \in \mathcal{V}\left(\mathcal{K}_{2}\left(\Omega_{\hat{x}}\right)\right)} \alpha_{2}(v) \epsilon \geq \sigma_{1}(\hat{x}) .
\end{aligned}
$$

Note that inclusion (13c) is obtained due to the convexity of $\sigma_{1}(\hat{x})$. Also, the right-hand inequality of (13c) becomes equality only if $\alpha_{2}(v)=0$ for all $v \in \mathcal{V}\left(\mathcal{K}_{2}\left(\Omega_{\hat{x}}\right)\right)$, leading to $\hat{x} \in \mathcal{K}_{1}\left(\Omega_{\hat{x}}\right)$. Therefore, $\ell_{2}(\hat{x})=\sigma_{1}(\hat{x})=\ell_{1}(\hat{x})$ for all $\hat{x} \in \mathcal{K}_{1}\left(\Omega_{\hat{x}}\right)$. Suppose claim 4) holds until $i$, leading to $h_{j} \geq \sigma_{i}(v)$ for all $v \in \mathcal{V}\left(\mathcal{K}_{j}\left(\Omega_{\hat{x}}\right)\right)$ and all $j \in \mathcal{I}_{i}$. We prove that it holds for $i+1$. In fact, following the same argument of use to prove $h_{2} \geq h_{1}+\epsilon$, we can obtain $h_{i+1} \geq \epsilon+h_{i}$. Accordingly, any point $\left[\hat{x}^{T} z\right]^{T} \in \Pi_{i+1}$ can be written in the following form:

$$
\begin{aligned}
& \alpha_{j}(v) \geq 0, \sum_{j=0}^{i+1} \sum_{v \in \mathcal{V}\left(\mathcal{K}_{j}\left(\Omega_{\hat{x}}\right)\right)} \alpha_{j}(v)=1 \\
& {\left[\hat{x}^{T} z\right]^{T}=\sum_{j=0}^{i+1} \sum_{v \in \mathcal{V}\left(\mathcal{K}_{j}\left(\Omega_{\hat{x}}\right)\right)} \alpha_{j}(v)\left[v^{T} h_{j}\right]^{T} .}
\end{aligned}
$$

As a consequence, the definition of $h_{i+1}$ in (11) yields:

$$
\begin{aligned}
z & =\sum_{j=0}^{i+1} \sum_{v \in \mathcal{V}\left(\mathcal{K}_{j}\left(\Omega_{\hat{x}}\right)\right)} \alpha_{j}(v) h_{j} \\
& \geq \sum_{j=0}^{i+1} \sum_{v \in \mathcal{V}\left(\mathcal{K}_{j}\left(\Omega_{\hat{x}}\right)\right)} \alpha_{j}(v) \sigma_{i}(v)+\sum_{v \in \mathcal{V}\left(\mathcal{K}_{i+1}\left(\Omega_{\hat{x}}\right)\right)} \alpha_{i+1}(v) \epsilon \\
& \geq \sigma_{i}(\hat{x})+\sum_{v \in \mathcal{V}\left(\mathcal{K}_{i+1}\left(\Omega_{\hat{x}}\right)\right)} \alpha_{i+1}(v) \epsilon \geq \sigma_{i}(\hat{x}) .
\end{aligned}
$$

Note that the left-hand inequality of $(15 b)$ is due to the convexity of $\sigma_{i}(\hat{x})$. The equality holds true only if $\alpha_{i+1}(v)=$ 0 for all $v \in \mathcal{V}\left(\mathcal{K}_{i+1}\left(\Omega_{\hat{x}}\right)\right)$, leading to $\hat{x} \in \mathcal{K}_{i}\left(\Omega_{\hat{x}}\right)$. In other words, $\ell_{i+1}(\hat{x})=\sigma_{i}(\hat{x})=\ell_{i}(\hat{x})$ for $\hat{x} \in \mathcal{K}_{i}\left(\Omega_{\hat{x}}\right)$.

\section{RobUST OUTPUT FEEDBACK CONTROL DESIGN}

This section presents the main result of this paper, i.e., a control design procedure based on the convex lifting $\ell_{N}(\hat{x})$, constructed in the preceding section. This procedure is summarized in Algorithm 1.

Algorithm 1 Control design procedure

Input: A local controller $u=K \hat{x}$ associated with $\Omega_{\hat{x}}$, $\ell_{N}(\hat{x})$ and $\mathcal{K}_{N}\left(\Omega_{\hat{x}}\right)$.

Output: optimal controller $u^{*}\left(\hat{x}_{k}\right)$ at each instant.

1: Compute $\ell_{N}\left(\hat{x}_{k}\right)$.

2: If $\hat{x}_{k} \in \Omega_{\hat{x}}$, then $u^{*}\left(\hat{x}_{k}\right)=K \hat{x}_{k}$.

3: Else Solve the following problem:

$$
\begin{gathered}
{\left[\gamma^{*}\left(u_{k}^{*}\right)^{T}\right]^{T}=\arg \min _{\gamma, u_{k}} \gamma} \\
\text { s.t. } \ell_{N}\left(A \hat{x}_{k}+B u_{k}+w\right) \leq \gamma \ell_{N}\left(\hat{x}_{k}\right), \\
\left(A \hat{x}_{k}+B u_{k}\right) \oplus\left(L \mathbb{V} \oplus L C \Omega_{e}\right) \subseteq \mathcal{K}_{N}\left(\Omega_{\hat{x}}\right), \\
u_{k} \in \mathbb{U}, \gamma \geq 0, \forall w \in \mathcal{V}\left(L \mathbb{V} \oplus L C \Omega_{e}\right) .
\end{gathered}
$$

4: $u^{*}\left(\hat{x}_{k}\right)=u_{k}^{*}$.

5: End

6: $k \leftarrow k+1$. Return to step 1 .

We now need to prove that the controller designed in Algorithm 1 can both guarantee the recursive feasibility and closed-loop stability of system (5), while still satisfying constraints (6). For ease of presentation, we first prove that the above controller makes function $\ell_{N}\left(\hat{x}_{k}\right)$ strictly decreasing along the closed-loop dynamics outside $\Omega_{\hat{x}}$. This observation is formally stated in the following result.

Proposition 4.1: Consider controller designed in Algorithm 1 and function $\ell_{N}(\hat{x})$, then for any $\hat{x} \in \mathcal{K}_{N}\left(\Omega_{\hat{x}}\right) \backslash \Omega_{\hat{x}}$, it follows that

$\ell_{N}\left(A \hat{x}+B u^{*}(\hat{x})+w\right)<\ell_{N}(\hat{x})$ for all $w \in L \mathbb{V} \oplus L C \Omega_{e}$. Proof: First, we will prove that any $v \in$ $\bigcup_{i \in \mathcal{I}_{N}} \mathcal{V}\left(\mathcal{K}_{i}\left(\Omega_{\hat{x}}\right)\right) \backslash \Omega_{\hat{x}}$ satisfies $\ell_{N}\left(A v+B u^{*}(v)+w\right)<$ $\ell_{N}(v)$ for all $w \in L \mathbb{V} \oplus L C \Omega_{e}$. In fact, for ease of presentation, define the following index, with respect to $\hat{x} \in \mathcal{K}_{N}\left(\Omega_{\hat{x}}\right), i^{*}(\hat{x})=\min _{j \in \mathcal{I}_{N} \cup\{0\}} j$ s.t. $\hat{x} \in \mathcal{K}_{j}\left(\Omega_{\hat{x}}\right)$. Obviously, for an $\hat{x} \in \mathcal{K}_{N}\left(\Omega_{\hat{x}}\right)$, the definition of $i^{*}(\hat{x})$ is unique. According to claim 2) of Lemma 3.5, for any $v \in \bigcup_{i \in \mathcal{I}_{N}} \mathcal{V}\left(\mathcal{K}_{i}\left(\Omega_{\hat{x}}\right)\right) \backslash \Omega_{\hat{x}}$, it yields

$$
\ell_{N}(v)=h_{i^{*}(v)}>0, i^{*}(v) \geq 1 .
$$

Also, there exists a control law $u(v) \in \mathbb{U}$ such that $A v+$ $B u(v)+w \in \mathcal{K}_{i^{*}(v)-1}\left(\Omega_{\hat{x}}\right)$, leading to

$$
\ell_{N}(A v+B u(v)+w) \leq h_{i^{*}(v)-1}, \forall w \in L \mathbb{V} \oplus L C \Omega_{e},
$$

as shown in claim 3) of Lemma 3.5. As a consequence of claim 1) in Lemma 3.5, inclusions (17) and (18) lead to

$$
\ell_{N}(A v+B u(v)+w) \leq h_{i^{*}(v)-1}<h_{i^{*}(v)}=\ell_{N}(v) .
$$

Otherwise, if $v \in \mathcal{V}\left(\Omega_{\hat{x}}\right)$, then there also exists a controller $u(v)=K v$ such that $A v+B u(v)+w \in \Omega_{\hat{x}}$ for all $w \in$ 
$L \mathbb{V} \oplus L C \Omega_{e}$, because $\Omega_{\hat{x}}$ is a robust positively invariant set. Therefore, claim 4) of Lemma 3.5 and claim 2) of Lemma 3.2 lead to

$$
\ell_{N}(A v+B u(v)+w)=\ell_{N}(v)=0 .
$$

Consider any point $\hat{x} \in \mathcal{K}_{N}\left(\Omega_{\hat{x}}\right) \backslash \Omega_{\hat{x}}$, there exists a region $\mathcal{X}_{j}^{(N)}$ in the polytopic partition $\left\{\mathcal{X}_{j}^{(N)}\right\}_{j \in \mathcal{I}_{M^{(N)}}}$ of $\mathcal{K}_{N}\left(\Omega_{\hat{x}}\right)$ such that $\hat{x} \in \mathcal{X}_{j}^{(N)}$. Accordingly, $\hat{x}$ can be expressed as:

$$
\hat{x}=\sum_{v \in \mathcal{V}\left(\mathcal{X}_{j}^{(N)}\right)} \alpha(v) v, \alpha(v) \geq 0, \sum_{v \in \mathcal{V}\left(\mathcal{X}_{j}^{(N)}\right)} \alpha(v)=1 .
$$

This yields

$$
\begin{aligned}
\ell_{N}(\hat{x}) & =\sum_{v \in \mathcal{V}\left(\mathcal{X}_{j}^{(N)}\right)} \alpha(v) \ell_{N}(v) \\
& >\sum_{v \in \mathcal{V}\left(\mathcal{X}_{j}^{(N)}\right)} \alpha(v) \ell_{N}(A v+B u(v)+w) \\
& \geq \ell_{N}\left(A \hat{x}+B \sum_{v \in \mathcal{V}\left(\mathcal{X}_{j}^{(N)}\right)} \alpha(v) u(v)+w\right) \\
& \geq \ell_{N}\left(A \hat{x}+B u^{*}(\hat{x})+w\right) .
\end{aligned}
$$

Note that equality (22a) holds because $\ell_{N}(\hat{x})$ is affine over $\mathcal{X}_{j}^{(N)}$. Since $\hat{x} \in \mathcal{K}_{N}\left(\Omega_{\hat{x}}\right) \backslash \Omega_{\hat{x}}$, there exists at least one vertex $v \in \mathcal{V}\left(\mathcal{X}_{j}^{(N)}\right) \backslash \Omega_{\hat{x}}$ such that $\alpha(v)>0$. Accordingly, inclusions (19) and (20) lead to the strict inequality (22b). Moreover, inclusion (22c) holds due to the convexity of $\ell_{N}(\hat{x})$. Finally, inequality (22d) is obtained because of $u(v) \in \mathbb{U}$ for all $v \in \mathcal{V}\left(\mathcal{X}_{j}^{(N)}\right)$ and the convexity of $\mathbb{U}$. The proof is complete.

Proposition 4.2: Consider controller designed in Algorithm 1 and function $\ell_{N}(\hat{x})$, then for any $\hat{x} \in \Omega_{\hat{x}}$, it yields

$\ell_{N}\left(A \hat{x}+B u^{*}(\hat{x})+w\right)=\ell_{N}(\hat{x})=0, \forall w \in L \mathbb{V} \oplus L C \Omega_{e}$.

Proof: Due to the robust positive invariance of $\Omega_{\hat{x}}$, for any $\hat{x} \in \Omega_{\hat{x}}$, it yields for all $w \in L \mathbb{V} \oplus L C \Omega_{e}$,

$$
A \hat{x}+B u^{*}(\hat{x})+w=(A+B K) \hat{x}+w \in \Omega_{\hat{x}} .
$$

Accordingly, claim 2) of Lemma 3.2 leads to

$$
\ell_{N}\left(A \hat{x}+B u^{*}(\hat{x})+w\right)=0, \forall w \in L \mathbb{V} \oplus L C \Omega_{e} .
$$

The proof is complete.

The main results of the paper are presented in the sequel.

Theorem 4.3: Consider system (5) subject to constraints (6). Then, for any initial point $\hat{x}_{0} \in \mathcal{K}_{N}\left(\Omega_{\hat{x}}\right)$, controller designed in Algorithm 1 guarantees the recursive feasibility and robust stability.

Proof: The recursive feasibility is guaranteed by suitable $\gamma<1$ according to Propositions 4.1 and 4.2. Moreover, Proposition 4.1 shows that $\ell_{N}\left(\hat{x}_{k}\right)$ is strictly decreasing along the closed-loop dynamics outside $\Omega_{\hat{x}}$, it is also bounded in $\left[0, h_{N}\right]$, therefore $\left\{\ell_{N}\left(\hat{x}_{k}\right)\right\}_{k=0}^{\infty}$ is convergent to 0 . As a consequence, $\hat{x}_{k}$ converges to $\Omega_{\hat{x}}$ as time tends to infinity, leading to robust stability of system (5).
Theorem 4.4: Consider system (1) subject to constraints (2). If the initial points $e_{0} \in \Omega_{e}$ and $\hat{x}_{0} \in \mathcal{K}_{N}\left(\Omega_{\hat{x}}\right)$, then controller designed in Algorithm 1 also guarantees the recursive feasibility and robust stability.

Proof: As $\Omega_{e}$ is a robust positively invariant set of system (4), then $e_{k} \in \Omega_{e}$, when $e_{0} \in \Omega_{e}$. Also, as shown in Theorem 4.3, controller designed in Algorithm 1 also guarantees the recursive feasibility for system (5), therefore $\hat{x}_{k} \in \mathbb{X} \ominus \Omega_{e}$. As a consequence, $x_{k}=e_{k}+\hat{x}_{k} \in \mathbb{X}$. Moreover, this controller satisfies the constraints in (2), leading to the recursive feasibility for system (1).

As for robust stability of system (1) and controller designed in Algorithm 1, it can be observed that $\hat{x}_{k}$ converges to $\Omega_{\hat{x}}$, while $e_{k}$ is bounded in $\Omega_{e}$, therefore $x_{k}=e_{k}+\hat{x}_{k}$ is convergent to $\Omega_{\hat{x}} \oplus \Omega_{e}$ as time tends to infinity, leading to its robust stability.

Recall that the vertex control in [10] and the one in [4] cannot guarantee that the closed-loop dynamics converge to a given robust positively invariant set, i.e., the result in Proposition 4.1. Note also that the construction of $\ell_{N}(\hat{x})$ becomes more demanding when the dimension of the state space increases, since it requires vertex enumeration. However, as this construction is performed offline, it is reasonable to assume that powerful computational resources are available.

\section{Numerical EXAMPLE}

In this section, a numerical example is considered to illustrate the proposed robust output feedback control design procedure. To this end, a DC-DC converter model is employed and given below:

$$
\begin{aligned}
x_{k+1} & =\left[\begin{array}{cc}
1 & 0.0075 \\
-0.143 & 0.996
\end{array}\right] x_{k}+\left[\begin{array}{c}
4.798 \\
0.115
\end{array}\right] u_{k}+w_{k} \\
y_{k} & =\left[\begin{array}{ll}
-0.5 & 1
\end{array}\right] x_{k}+v_{k} .
\end{aligned}
$$

Also, the state, control variable and disturbances satisfy:

$$
\begin{gathered}
-\left[\begin{array}{ll}
10 & 10
\end{array}\right]^{T} \leq x_{k} \leq\left[\begin{array}{ll}
3 & 3
\end{array}\right]^{T},\left\|u_{k}\right\|_{\infty} \leq 0.5 \\
\left\|w_{k}\right\|_{\infty} \leq 0.01,\left\|v_{k}\right\|_{\infty} \leq 0.005 .
\end{gathered}
$$

We choose the observer gain $L=[-0.56400 .4838]$ and a local controller $u=[-0.23130 .1781] \hat{x}$. Accordingly, the minimal robust positively invariant set $\Omega_{e}$ is computed, using the algorithm presented in [25], to enlarge the feasible region for the observer system (3). Also, $\Omega_{\hat{x}}$ is chosen as the maximal output admissible set associated with the local controller $u=\left[\begin{array}{ll}-0.2313 & 0.1781\end{array}\right] \hat{x}$. The set $\Omega_{\hat{x}} \oplus \Omega_{e}$ and the 20 -step controllable set $\mathcal{K}_{20}\left(\Omega_{\hat{x}}\right) \oplus \Omega_{e}$ are both represented in Fig. 1. Subsequently, a convex lifting $\ell_{20}(\hat{x})$ is constructed following the construction in (7) and (11) with $h_{1}=10^{-2}$ and $\epsilon=10^{-3}$. This convex lifting is presented in Fig. 2 . Also, it is shown in this figure the evolution of $\ell_{20}\left(\hat{x}_{k}\right)$ along the closed-loop dynamics of system (5). It verifies that the convex lifting $\ell_{20}\left(\hat{x}_{k}\right)$ is strictly decreasing along the dynamics outside $\Omega_{\hat{x}}$, leading to the convergence of $\ell_{20}\left(\hat{x}_{k}\right)$ to 0 . Roughly speaking, $x_{k}$ converges to $\Omega_{\hat{x}} \oplus \Omega_{e}$ as shown more clearly in Fig. 1. Finally, the numerical example of this paper is simulated in the environment of MPT 3.0 [11]. 


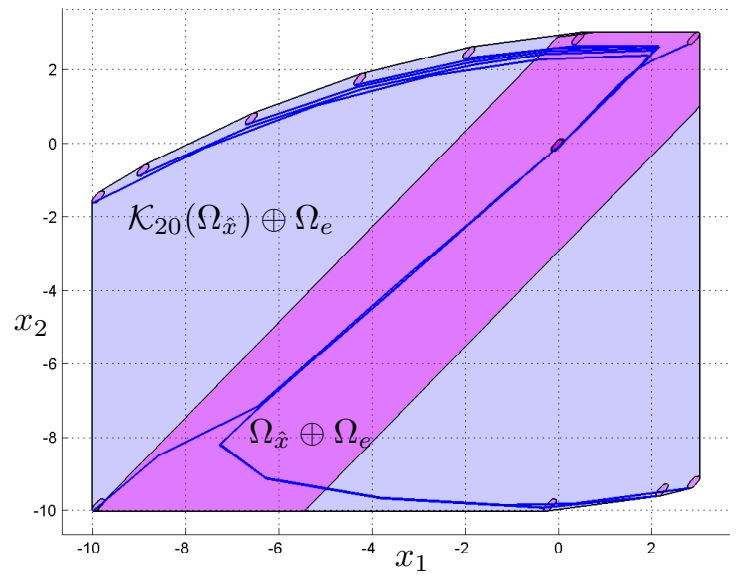

Fig. 1. Maximal output admissible set $\Omega_{\hat{x}} \oplus \Omega_{e}$, the 20-step controllable set $\mathcal{K}_{20}\left(\Omega_{\hat{x}}\right) \oplus \Omega_{e}$ and the closed-loop dynamics of system (1).

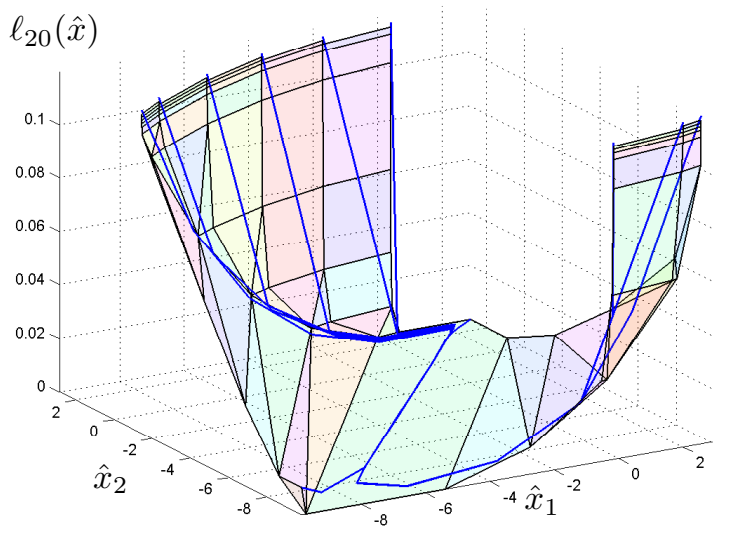

Fig. 2. The constructed convex lifting $\ell_{20}(\hat{x})$ over $\mathcal{K}_{20}\left(\Omega_{\hat{x}}\right)$ with $h_{1}=$ $10^{-2}$ and $\epsilon=10^{-3}$ and its strict decrease along the closed-loop dynamics of the observer system (3) outside $\Omega_{\hat{x}}$.

\section{CONCLUSIONS}

This paper presented a method for robust output feedback control of linear systems affected by both state and output additive disturbances. The method made use of a suitable convex lifting, which was shown to be strictly decreasing along the closed-loop dynamics outside a given robust positively invariant set. Accordingly, the closed-loop dynamics were shown to be convergent to this invariant set, leading to robust stability. The method was shown to only require solving a linear program at each sampling instant. Finally, a numerical example was considered to illustrate the method.

\section{REFERENCES}

[1] G. Bitsoris, "On the positive invariance of polyhedral sets for discretetime systems," Systems \& Control Letters, vol. 11, no. 3, pp. 243-248, 1988.

[2] — - "Positively invariant polyhedral sets of discrete-time linear systems," International Journal of Control, vol. 47, no. 6, pp. 17131726, 1988.

[3] G. Bitsoris and M. Vassilaki, "Constrained regulation of linear systems," Automatica, vol. 31, no. 2, pp. 223-227, 1995.

[4] F. Blanchini, "Ultimate boundedness control for uncertain discretetime systems via set-induced Lyapunov functions," Automatic Control, IEEE Transactions on, vol. 39, no. 2, pp. 428-433, Feb 1994.
[5] F. Blanchini and S. Miani, Set-theoretic methods in control. Springer, 2007.

[6] R. Findeisen, L. Imsland, F. Allgower, and B. A. Foss, "State and output feedback nonlinear model predictive control: An overview," European journal of control, vol. 9, no. 2, pp. 190-206, 2003.

[7] T. Gal, Postoptimal analyses, parametric programming and related topics. Walter de Gruyter, 1995.

[8] E. G. Gilbert and K. T. Tan, "Linear systems with state and control constraints: The theory and application of maximal output admissible sets," Automatic Control, IEEE Transactions on, vol. 36, no. 9, pp. 1008-1020, 1991.

[9] M. Gulan, G. Takács, N. A. Nguyen, S. Olaru, P. Rodriguez-Ayerbe, and B. Roha-Ilkiv, "Embedded linear model predictive control for 8-bit microcontrollers via convex lifting," in the 20th IFAC World Congress, Toulouse, France, July 9-14 2017.

[10] P.-O. Gutman and M. Cwikel, "An algorithm to find maximal state constraint sets for discrete-time linear dynamical systems with bounded controls and states," Automatic Control, IEEE Transactions on, vol. 32 no. 3, pp. 251-254, 1987.

[11] M. Herceg, M. Kvasnica, C. Jones, and M. Morari, "Multi-parametric toolbox 3.0," in Control Conference (ECC), 2013 European, July 2013, pp. $502-510$.

[12] E. C. Kerrigan, "Robust constraint satisfaction: Invariant sets and predictive control," Ph.D. dissertation, University of Cambridge, 2001.

[13] I. Kolmanovsky and E. G. Gilbert, "Theory and computation of disturbance invariant sets for discrete-time linear systems," Mathematical Problems in Engineering, vol. 4, no. 4, pp. 317-367, 1998.

[14] D. Q. Mayne, S. V. Raković, R. Findeisen, and F. Allgöwer, "Robust output feedback model predictive control of constrained linear systems," Automatica, vol. 42, no. 7, pp. 1217-1222, 2006.

[15] D. Q. Mayne, J. B. Rawlings, C. V. Rao, and P. O. M. Scokaert, "Constrained model predictive control: Stability and optimality," $\mathrm{Au}$ tomatica, vol. 36, no. 6, pp. 789-814, 2000.

[16] M. Morari and E. Zafiriou, Robust process control. Prentice hall Englewood Cliffs, NJ, 1989, vol. 488.

[17] H.-N. Nguyen, P.-O. Gutman, S. Olaru, and M. Hovd, "Implicit improved vertex control for uncertain, time-varying linear discretetime systems with state and control constraints," Automatica, vol. 49 , no. 9, pp. 2754-2759, 2013.

[18] H.-N. Nguyen, S. Olaru, P. O. Gutman, and M. Hovd, "Constrained control of uncertain, time-varying linear discrete-time systems subject to bounded disturbances," IEEE Transactions on Automatic Control, vol. 60 , no. 3, pp. 831-836, 2015.

[19] N. A. Nguyen, M. Gulan, S. Olaru, and P. Rodriguez-Ayerbe, "Convex lifting: Theory and control applications," Automatic Control, IEEE Transactions on, 2017. [Online]. Available: https://halcentralesupelec.archives-ouvertes.fr/hal-01326804/document

[20] N. A. Nguyen, S. Olaru, and P. Rodriguez-Ayerbe, "Any discontinuous PWA function is optimal solution to a parametric linear programming problem," in 54th IEEE Conference on Decision and Control, Osaka, Japan, 2015.

[21] —, "Inverse parametric linear/quadratic programming problem for continuous PWA functions defined on polyhedral partitions of polyhedra," in 54th IEEE Conference on Decision and Control, Osaka, Japan, 2015.

[22] - "Robust control design based on convex liftings," in the 8th IFAC Symposium on Robust Control Design, 2015.

[23] N. A. Nguyen, S. Olaru, P. Rodriguez-Ayerbe, M. Hovd, and I. Necoara, "Constructive solution of inverse parametric linear/quadratic programming problems," Journal of Optimization Theory and Applications, vol. 172, no. 2, pp. 623-648, 2017.

[24] N. A. Nguyen, S. Olaru, P. Rodríguez-Ayerbe, and M. Kvasnica, "Convex liftings-based robust control design," Automatica, vol. 77, pp. $206-213,2017$

[25] S. V. Rakovic, E. C. Kerrigan, K. I. Kouramas, and D. Q. Mayne, "Invariant approximations of the minimal robust positively invariant set," Automatic Control, IEEE Transactions on, vol. 50, no. 3, pp. 406-410, 2005

[26] D. Sui, L. Feng, and M. Hovd, "Robust output feedback model predictive control for linear systems via moving horizon estimation," in 2008 American Control Conference. IEEE, 2008, pp. 453-458.

[27] K. Zhou and J. C. Doyle, Essentials of robust control. Prentice hall Upper Saddle River, NJ, 1998, vol. 104. 